\title{
Point-of-care ultrasound in undergraduate medical education: an opportunity for anesthesiologists
}

\author{
Sabrina Alani, BSc $\cdot$ Michelle Moody, BN RN
}

Received: 23 April 2018/Revised: 14 June 2018/Accepted: 25 June 2018/Published online: 7 August 2018

(c) Canadian Anesthesiologists' Society 2018

\section{To the Editor,}

Anesthesiologists have a uniquely broad spectrum of practice and deliver a wide variety of clinical services. They are considered to have expertise in numerous fields, including but not limited to cardiology, respirology, pharmacology, airway management, and critical care medicine. As such, they are well-suited to educating undergraduate medical students on any number of subjects.

As medical students, we have identified an existing gap in our education to which anesthesiologists would be ideally suited to respond. Point-of-care ultrasound (POCUS) is in its preliminary stages of introduction in multiple Canadian undergraduate medical education (UGME) programs. A number of studies have identified the value of integrating POCUS in a longitudinal manner, with an initial introduction in the pre-clerkship component of UGME. Medical students are capable of learning ultrasound skills. These skills enhance and reinforce the education of basic science topics, such as anatomy, physiology, and pathology, and also act as a "natural bridge from basic science to clinical science." ${ }^{1,2}$ The trend of modern-day medicine is such that the skill of bedside ultrasound is as fundamental to physicians as the use of a stethoscope. ${ }^{1}$ In line with this trend, schools are now beginning to create ultrasound curricula for their undergraduate programs. Some studies have shown that the integration of online teaching modules and simulation

S. Alani, BSc $\cdot$ M. Moody, BN RN

Faculty of Medicine, Memorial University of Newfoundland,

St. John's, NL, Canada

S. Alani, BSc · M. Moody, BN RN ( $\square)$

Health Sciences Centre, St. John's, NL, Canada

e-mail: mam524@mun.ca is the best approach to introducing a core curriculum of POCUS training. ${ }^{3,4}$ As pioneers in the use of simulation for medical education, anesthesiologists are uniquely poised to provide simulation-based instruction to medical students. This new curriculum provides a unique opportunity for anesthesiologists to not only increase their participation in UGME, but also to present themselves as leaders in the field of POCUS.

Despite the expertise that anesthesiologists have to offer, they have had limited engagement in the UGME curriculum. ${ }^{5}$ A 2017 study showed that in the majority of Canadian undergraduate programs, anesthesiologists spent significantly fewer hours teaching the UGME curriculum than expected based on their proportional faculty size; 6 of 17 departments contributed less than $1 \%$ of pre-clerkship hours. ${ }^{5}$ The study went on to suggest that the majority of teaching from anesthesiologists in the undergraduate setting is provided by a small number of dedicated teachers.

Despite relatively low participation in UGME, anesthesiologists often view themselves as indispensable to medical education. Nevertheless, UGME program directors frequently view their areas of expertise as topics that can be taught by other medical professionals - and even non-medical professionals. When associate deans discuss the same topic, they identify numerous areas where anesthesiology could contribute to pre-clerkship education. POCUS represents a new opportunity for anesthesiologists to interact with undergraduates and highlight all that the specialty has to offer. ${ }^{5}$

The cornerstones of any rewarding medical profession include the delivery of innovative clinical care, engagement in medical education, and creation of new knowledge through research. ${ }^{5}$ Specialists who share anesthesiologists' interests in trauma and critical care, 
such as emergency room physicians and intensivists, offer partnership opportunities to get anesthesiologists involved in the development or refinement of the POCUS undergraduate curriculum. If anesthesiologists fail to take a leadership role in the teaching of POCUS in UGME they, and their specialty, will lose a rare opportunity to influence students and future colleagues.

Conflicts of interest None declared.

Editorial responsibility This submission was handled by Dr. Gregory L. Bryson, Deputy Editor-in-Chief, Canadian Journal of Anesthesia.

\section{References}

1. Palma JK. Successful strategies for integrating bedside ultrasound into undergraduate medical education. Mil Med 2015; 180: 153-7.

2. Stansfield E, Woo MY, Tam R, Pugh D, McInnes M, Hamstra SJ. Designing a multi-disciplinary undergraduate medical school ultrasonography curriculum. UOJM 2014; 4: 49-54.

3. Meineri M, Bryson GL, Arellano R, Skubas N. Core point-of-care ultrasound curriculum: what does every anesthesiologist need to know? Can J Anesth 2018; 65: 417-26.

4. Mitchell JD, Montealegre-Gallegos M, Mahmood $F$, et al. Multimodal perioperative ultrasound course for interns allows for enhanced acquisition and retention of skills and knowledge. A A Case Rep 2015; 5: 119-23.

5. Hamlin $C$, Bhangu K, Villafranca A, et al. Participation of Canadian anesthesiology departments in undergraduate medical education. Can J Anesth 2017; 64: 16-28. 